\title{
Lattice Methods for Pricing American Strangles with Two-Dimensional Stochastic Volatility Models
}

\author{
Xuemei Gao, ${ }^{1}$ Dongya Deng, ${ }^{2}$ and Yue Shan ${ }^{2}$ \\ ${ }^{1}$ School of Economic Mathematics and School of Finance, Southwestern University of Finance and Economics, Chengdu 611130, China \\ ${ }^{2}$ School of Finance, Southwestern University of Finance and Economics, Chengdu 611130, China \\ Correspondence should be addressed to Dongya Deng; 112020204015@2012.swufe.edu.cn
}

Received 22 January 2014; Accepted 5 April 2014; Published 28 April 2014

Academic Editor: Chuangxia Huang

Copyright (c) 2014 Xuemei Gao et al. This is an open access article distributed under the Creative Commons Attribution License, which permits unrestricted use, distribution, and reproduction in any medium, provided the original work is properly cited.

\begin{abstract}
The aim of this paper is to extend the lattice method proposed by Ritchken and Trevor (1999) for pricing American options with one-dimensional stochastic volatility models to the two-dimensional cases with strangle payoff. This proposed method is compared with the least square Monte-Carlo method via numerical examples.
\end{abstract}

\section{Introduction}

Calculating American style options under geometric Brownian motion is far from the realistic financial market. It is more valuable to price American style options under stochastic models. In general the valuation of American options with stochastic volatility models has no closed-form solution except very few cases (see, e.g., Heston [1]). Therefore numerical methods or simulation methods are developed to price financial derivatives with stochastic volatility, among which the lattice methods receive much more attention. Ritchken and Trevor [2] proposed an efficient lattice method for pricing American options under GARCH models. Later the idea was further developed and applied by several papers, for example, Cakici and Topyan [3] and Wu [4], and recently the convergence of the method was proved by Akyildirim et al. [5].

All the abovementioned references focused on the development of lattice methods for pricing American options with one underlying asset and single stochastic volatility model. To the best of our knowledge, there are no papers studying the lattice methods for options with many underlying assets and multidimensional stochastic volatility models. Indeed there are many papers in developing lattice methods for pricing options with many underlying assets, for example, Boyle [6], Boyle et al. [7], Chen et al. [8], Gamba and Trigeorgis [9], and Moon et al. [10]. However it is not seen for lattice methods for multidimensional stochastic volatility models.
In this paper we give an attempt to this challenging topic by studying an American style option with strangle payoff, which was previously investigated by Chiarella and Ziogas [11] and Moraux [12] for single asset and constant volatility. We develop the lattice methods of Ritchken and Trevor [2] to the American strangle options with many underlying assets and multidimensional stochastic volatility GARCH models. We compare the lattice methods with the least square MonteCarlo methods via several numerical examples.

\section{Two-Dimensional Stochastic Volatility Models of American Strangles}

Assume that the prices of two-dimensional assets $\mathbf{S}_{\mathbf{t}}=$ $\left(S_{t}^{1}, S_{t}^{2}\right)^{T}$ follow a two-dimensional GARCH model (see, e.g., Duan [13] for more explanation of the one-dimensional GARCH model). Consider

$$
\begin{gathered}
\ln \left(\frac{S_{t+1}^{i}}{S_{t}^{i}}\right)=r_{f}-q^{i}+\lambda_{i} \sqrt{h_{t}^{i}}-\frac{1}{2} h_{t}^{i}+\sqrt{h_{t}^{i}} v_{t+1}^{i}, \\
h_{t+1}^{i}=\beta_{0}^{i}+\beta_{1}^{i} h_{t}^{i}+\beta_{2}^{i} h_{t}^{i}\left(v_{t+1}^{i}-c^{i}\right)^{2},
\end{gathered}
$$

with $i=1,2$, where $S_{t}^{i}$ is the price of the $i$ th asset corresponding to the standard Brown motion, $q^{i}$ is the dividend rate for the $i$ th asset, $h_{t}^{i}$ is the volatility of the $i$ th asset price, $v_{t+1}^{i}$, conditional on information at time $t$, is a standard normal 
random variable, $r_{f}$ is the riskless rate of return over the period, and $\lambda_{i}$ is the unit risk premium for the $i$ th asset. Under the local risk-neutralized measure, the processes (1) are written as

$$
\begin{aligned}
& \ln \left(\frac{S_{t+1}^{i}}{S_{t}^{i}}\right)=r_{f}-q^{i}-\frac{1}{2} h_{t}^{i}+\sqrt{h_{t}^{i} \mathbb{v}_{t+1}^{i},} \\
& h_{t+1}^{i}=\beta_{0}^{i}+\beta_{1}^{i} h_{t}^{i}+\beta_{2}^{i} h_{t}^{i}\left(\mathbb{v}_{t+1}^{i}-c^{*, i}\right)^{2},
\end{aligned}
$$

with $i=1,2$, where $v_{t+1}^{i}$, conditional on time $t$ information, is a standard normal random variable with respect to the riskneutralized measure, the parameters $\beta_{0}^{i}, \beta_{1}^{i}, \beta_{2}^{i}, c^{*, i}$ in the model can be obtained by regression on the financial market, and $h_{0}^{i}$ is the initial variance of asset $i$. Let $f\left(\mathbf{S}_{\mathbf{t}}\right)=\max \left(S_{t}^{1}, S_{t}^{2}\right)$ be a single-valued function of $\mathbf{S}_{\mathbf{t}}$. In this paper, we consider a two-dimensional assets American strangle option whose payoff at maturity $T$ is defined by

$$
\max \left(\left[K_{1}-f\left(\mathbf{S}_{\mathrm{T}}\right)\right]^{+},\left[f\left(\mathbf{S}_{\mathrm{T}}\right)-K_{2}\right]^{+}\right),
$$

in which $K_{1}$ and $K_{2}$, the strikes for American strangle's call and put parts, satisfy $K_{1}<K_{2}$.

\section{Lattice Algorithms}

Ritchken and Trevor [2] investigated the stochastic lattice methods for one-dimensional GARCH model. This paper intends to extend the methods to two-dimensional GARCH model. The aim of this paper is to design an algorithm that avoids an exponentially exploding number of states. Toward this goal, we begin by approximating the sequence of single period log normal random variables in (2) by a sequence of discrete random variables. In particular, assume the information set at date $t$ is $\left(S_{t}^{i}, h_{t}^{i}\right), i=1,2$, and let $y_{t}^{i}=\ln \left(S_{t}^{i}\right), i=1,2$. Then, viewed from date $t, y_{t+1}^{i}, i=$ 1,2 , are normal random variables with conditional moments. Consider

$$
\begin{gathered}
E_{t}\left[y_{t+1}^{i}\right]=y_{t}^{i}+r_{f}-q^{i}-\frac{1}{2} h_{t}^{i}, \\
\operatorname{Var}_{t}\left[y_{t+1}^{i}\right]=h_{t}^{i} .
\end{gathered}
$$

We establish two discrete state Markov chains' approximation, $\left(y_{a, t}^{i}, h_{a, t}^{i}\right), i=1,2$, for the dynamics of the discrete time state variables that converge to the continuous state $\left(y_{t}^{i}, h_{t}^{i}\right), i=1,2$. In particular, we approximate the sequence of conditional normal random variables by a sequence of discrete random variables. Given this period's logarithmic price and conditional variance, the conditional normal distribution of the next period's logarithmic price is approximated by a discrete random variable that takes on $2 n+1$ values for each asset. The lattice we construct has the property that the conditional means and variances of one period returns match the true means and variances given in (4), and the approximating sequence of discrete random variables converges to the true sequence of normal random variables. For each asset, the gap between adjacent logarithmic prices is determined by a spacing parameter $\gamma_{n}^{i}$ for the logarithmic returns in such a way that all the approximating logarithmic prices are separated by

$$
\gamma_{n}^{i}=\frac{\gamma^{i}}{\sqrt{n}}
$$

The size of these $2 n+1$ jumps is restricted to integer multiples of $\gamma_{n}^{i}$. Another important issue is to ensure valid probability values over the grid of $2 n+1$ prices; the size of these jumps needs to be adjusted accordingly. This is efficiently handled with the inclusion of a jump parameter $\eta^{i}$, which is an integer that depends on the level of the variance as follows:

$$
\eta^{i}-1<\frac{\sqrt{h_{a, t}^{i}}}{\gamma^{i}} \leq \eta^{i} .
$$

Consequently, the resulting two-asset GARCH model is

$$
\begin{gathered}
y_{a, t+1}^{i}=y_{a, t}^{i}+j \eta^{i} \gamma_{n}^{i}, \\
h_{a, t+1}^{i}=\beta_{0}^{i}+\beta_{1}^{i} h_{a, t}^{i}+\beta_{2}^{i} h_{a, t}^{i}\left(\varepsilon_{a, t+1}^{i}-c^{*, i}\right)^{2},
\end{gathered}
$$

for $i=1,2$, where

$$
\varepsilon_{a, t+1}^{i}=\frac{j \eta^{i} \gamma_{n}^{i}-\left(r_{f}-q^{i}-(1 / 2) h_{a, t}^{i}\right)}{\sqrt{h_{a, t}^{i}}}
$$

and $j=0, \pm 1, \pm 2, \ldots, \pm n, \gamma^{i}=\sqrt{h_{0}^{i}}, i=1,2$. The probability distribution for $y_{a, t+1}^{i}$, conditional on $y_{a, t}^{i}$ and $h_{a, t}^{i}$, is then given by

$\operatorname{Prob}\left(y_{a, t+1}^{i}=y_{a, t}^{i}+j \eta^{i} \gamma_{n}^{i}\right)=P^{i}(j), \quad j=0, \pm 1, \pm 2, \ldots, \pm n$,

where

$$
P^{i}(j)=\sum_{j_{u}, j_{m}, j_{d}}\left(\begin{array}{c}
n \\
j_{u} j_{m} j_{d}
\end{array}\right)\left(p_{u}^{i}\right)^{j_{u}}\left(p_{m}^{i}\right)^{j_{m}}\left(p_{d}^{i}\right)^{j_{d}}
$$

with $j_{u}, j_{m}, j_{d} \geq 0$ such that $n=j_{u}+j_{m}+j_{d}$ and $j=j_{u}-j_{d}$. Use the same lattice tree for assets $S_{1}$ and $S_{2}$ independently and assume each asset node has three possible paths to the next node: up, middle, and down. Then there are 9 possible combinations. The order of calculation is $\left(S_{1}^{\text {up }}, S_{2}^{\text {up }}\right),\left(S_{1}^{\text {up }}, S_{2}^{\text {middle }}\right),\left(S_{1}^{\text {up }}, S_{2}^{\text {down }}\right),\left(S_{1}^{\text {middle }}, S_{2}^{\text {up }}\right)$, $\left(S_{1}^{\text {middle }}, S_{2}^{\text {middle }}\right),\left(S_{1}^{\text {middle }}, S_{2}^{\text {down }}\right),\left(S_{1}^{\text {down }}, S_{2}^{\text {up }}\right),\left(S_{1}^{\text {down }}, S_{2}^{\text {middle }}\right)$, and $\left(S_{1}^{\text {down }}, S_{2}^{\text {down }}\right)$, which is illustrated by Figure 1 .

The possibilities for the nine combinations are $P^{1}(1) P^{2}(1), \quad P^{1}(1) P^{2}(0), \quad P^{1}(1) P^{2}(-1), \quad P^{1}(0) P^{2}(1)$, $P^{1}(0) P^{2}(0), P^{1}(0) P^{2}(-1), P^{1}(-1) P^{2}(1), P^{1}(-1) P^{2}(0)$, and $P^{1}(-1) P^{2}(-1)$. Then, the volatility pattern by restricting the storage of conditional variance to the minimum and maximum values at each node under the forward-building process needs to be constructed. At each node for each asset, the option prices over a grid of $K$ points are evaluated, covering the state space of the variances from the minimum to the maximum for each asset. Let $h_{a, t}^{i, \max }(m)$ and $h_{a, t}^{i, \min }(m)$ 
TABLE 1: Full volatility information at node $(t, m)$.

$$
\begin{gathered}
\hline h_{a, t}^{1}(3, m), h_{a, t}^{2}(3, m) \\
h_{a, t}^{1}(3, m), h_{a, t}^{2}(2, m) \\
h_{a, t}^{1}(3, m), h_{a, t}^{2}(1, m) \\
\hline h_{a, t}^{1}(2, m), h_{a, t}^{2}(3, m) \\
h_{a, t}^{1}(2, m), h_{a, t}^{2}(2, m) \\
h_{a, t}^{1}(2, m), h_{a, t}^{2}(1, m) \\
h_{a, t}^{1}(1, m), h_{a, t}^{2}(3, m) \\
h_{a, t}^{1}(1, m), h_{a, t}^{2}(2, m) \\
h_{a, t}^{1}(1, m), h_{a, t}^{2}(1, m) \\
\hline
\end{gathered}
$$

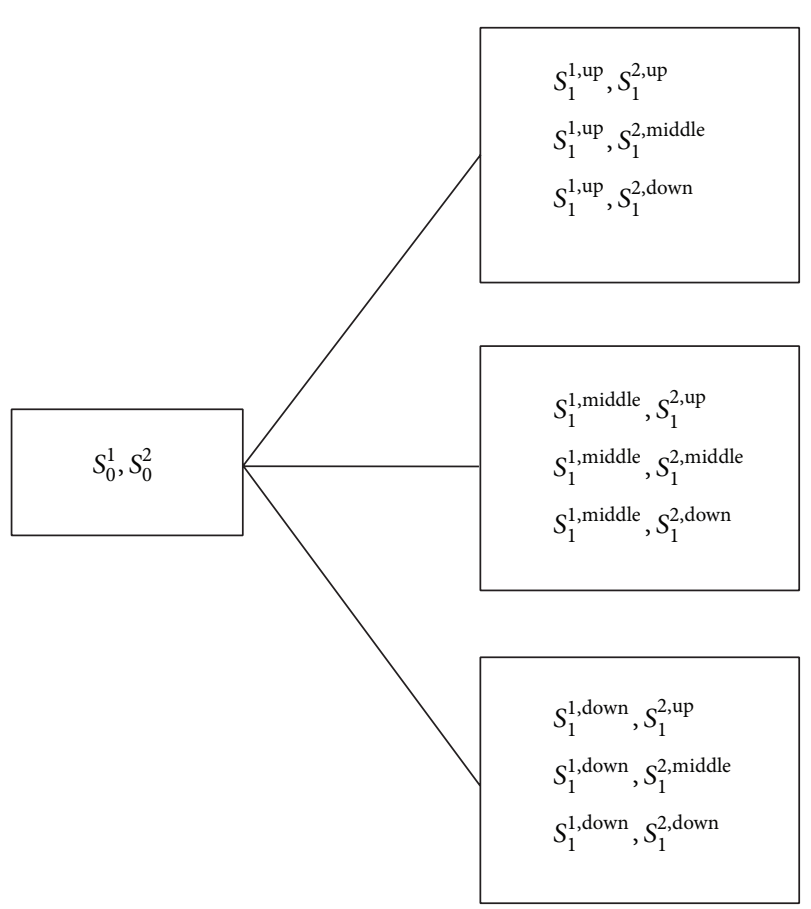

Figure 1: Two-asset GARCH tree.

represent the maximum and minimum variances that can be attained at node $m$ for asset $i$. Option prices at this node are computed for $K$ levels of variance ranging from the lowest to the highest at equidistant intervals. In particular, $h_{a, t}^{i}(k, m)$ representing the $k$ th level of the variance at node $(t, m)$ with $k=1, \ldots, K$ is defined by an interpolation as follows:

$$
h_{a, t}^{i}(k, m)=h_{a, t}^{i, \min }(m)+l_{t}^{i}(m)(k-1), \quad k=1, \ldots, K,
$$

where

$$
l_{t}^{i}(m)=\frac{h_{a, t}^{i, \max }(m)-h_{a, t}^{i, \min }(m)}{K-1} .
$$

For $K=3$, the full volatility information at node $(t, m)$ is described by Table 1 .
According to $\mathrm{Wu}[4]$, we have

$$
\begin{aligned}
p_{u}^{i}= & \frac{h_{a, t}^{i}}{2 n\left(\eta^{i} \gamma_{n}^{i}\right)^{2}}+\frac{r_{f}-q^{i}-(1 / 2) h_{a, t}^{i}}{2 n \eta^{i} \gamma_{n}^{i}} \\
& +\frac{\left(r_{f}-q^{i}-(1 / 2) h_{a, t}^{i}\right)^{2}}{2\left(n \eta^{i} \gamma_{n}^{i}\right)^{2}}, \\
p_{m}^{i}=1- & \frac{h_{a, t}^{i}}{n\left(\eta^{i} \gamma_{n}^{i}\right)^{2}}-\frac{\left(r_{f}-q^{i}-(1 / 2) h_{a, t}^{i}\right)^{2}}{\left(n \eta^{i} \gamma_{n}^{i}\right)^{2}} \\
p_{d}^{i}= & \frac{h_{a, t}^{i}}{2 n\left(\eta^{i} \gamma_{n}^{i}\right)^{2}}-\frac{r_{f}-q^{i}-(1 / 2) h_{a, t}^{i}}{2 n \eta^{i} \gamma_{n}^{i}} \\
& +\frac{\left(r_{f}-q^{i}-(1 / 2) h_{a, t}^{i}\right)^{2}}{2\left(n \eta^{i} \gamma_{n}^{i}\right)^{2}}
\end{aligned}
$$

where $i=1,2$ represent the $i$ asset, and $h_{a, t}^{i}$ is the approximation volatility of asset $i$ at time $t$, and $\eta^{i}$ is the jump parameter of asset $i$. Cakici and Topyan [3] modified the forward-building process and used interpolated variances only during the backward recursion to make the algorithm more efficient. They adopted only real node maximum and minimum variances, not the interpolated ones that fell between the maximum and minimum variances. It is intuitive to use interpolation for $K$ points in the backward procedure. At the terminal time $T$, the two-asset American strangle option's cash flow is

$$
\max \left\{\left[K_{1}-\max \left(S_{t}^{1}, S_{t}^{2}\right)\right]^{+},\left[\max \left(S_{t}^{1}, S_{t}^{2}\right)-K_{2}\right]^{+}\right\}
$$

Let $C_{a, t}(m, k)$ be the $k$ th option price at the node $(m, k)$, for $k=1,2, \ldots, K$, and the variance is $h_{a, t}^{i}(m, k), i=1,2$. Note that the boundary condition for a two-asset American strangle option with strike $X$ which expires in period $T$ is

$$
\begin{array}{r}
C_{a, T}(m, 1)=C_{a, T}(m, 2)=\cdots=C_{a, T}(m, K) \\
=\max \left\{\left[K_{1}-\max \left(S_{T}^{1}, S_{T}^{2}\right)\right]^{+},\right. \\
\left.\left[\max \left(S_{T}^{1}, S_{T}^{2}\right)-K_{2}\right]^{+}\right\} .
\end{array}
$$

We apply backward recursion to establish the option price at date 0 . Consider a node $(m, k)$ at time $t$. Then we compute the option price $C_{a, t}(m, k)$ corresponding to variance $h_{a, t}^{i}(m, k)$ at the node. Given the variance $h_{a, t}^{i}(m, k)$, we compute the appropriate jump parameter, $\eta^{i}$ for each asset, by (6). The successive nodes for this variance combination are $((t+1, m+$ $\left.\left.j^{1} \eta^{1}\right),\left(t+1, m+j^{2} \eta^{2}\right)\right)$, where $j^{1}=0, \pm 1, \pm 2, \ldots, \pm n$ and $j^{2}=0, \pm 1, \pm 2, \ldots, \pm n$. Equation (11) is used to compute the period $(t+1)$ variance for each of these nodes. Specifically, for the transition from the $k$ th variance element of node $(t, m)$ to 
node $\left(\left(t+1, m+j^{1} \eta^{1}\right),\left(t+1, m+j^{2} \eta^{2}\right)\right)$, the period $(t+1)$ variance for each asset is given by

$$
\begin{aligned}
h_{a, t+1}^{i, \text { next }}\left(j^{1,2}\right)= & \beta_{0}^{i}+\beta_{1}^{i} h_{a, t}^{i}(m, k) \\
& +\beta_{2}^{i} h_{a, t}^{i}(m, k)\left[\frac{\left(j \eta^{i} \gamma_{n}^{i}-r_{f}+h_{a, t}^{i}(m, k)\right)}{\sqrt{h_{a, t}^{i}(m, k)}-c^{i, *}}\right]^{2},
\end{aligned}
$$

where $j^{1,2}$ represents the combination of $j^{1}$ and $j^{2}$. Linear interpolation of the two stored option prices corresponding to the two stored variance entries closest to $h_{a, t+1}^{2 \text {,next }}\left(j^{2}\right)$ is used to obtain the option price corresponding to a variance of $h_{a, t+1}^{2, \text { next }}\left(j^{2}\right)$ when $h_{a, t+1}^{1, \text { next }}\left(j^{1}\right)$ is already chosen. Let $L$ be an integer smaller than $K$ defined via

$$
h_{a, t+1}^{2}\left(m+j^{2} \eta^{2}, L\right)<h_{a, t+1}^{2, \text { next }}\left(j^{2}\right)<h_{a, t+1}^{2}\left(m+j^{2} \eta^{2}, L+1\right) .
$$

The interpolated option price is

$$
\begin{aligned}
C^{\text {interp }}(m)= & q(j) C_{a, t+1}\left(m+j^{1,2} \eta^{1,2}, L\right) \\
& +(1-q(j)) C_{a, t+1}\left(m+j^{1,2} \eta^{1,2}, L+1\right),
\end{aligned}
$$

where

$$
q(j)=\frac{h_{a, t+1}^{2}\left(m+j^{2} \eta^{2}, L+1\right)-h_{a, t+1}^{2, \text { next }}\left(j^{2}\right)}{h_{a, t+1}^{2}\left(m+j^{2} \eta^{2}, L+1\right)-h_{a, t+1}^{2}\left(m+j^{2} \eta^{2}, L\right)} .
$$

In this way an option price is identified for each of the $(2 n+1)(2 n+1)$ jumps from node $(t, m)$ with variance combination $\left(h_{a, t+1}^{1, \text { next }}\left(j^{1}\right), h_{a, t+1}^{2, \text { next }}\left(j^{2}\right)\right)$. In each case, either node $\left(t+1, m+j^{1,2} \eta^{1,2}\right)$ contains a variance entry (and hence option value) that matches $\left(h_{a, t+1}^{1, \text { next }}\left(j^{1}\right), h_{a, t+1}^{2, \text { next }}\left(j^{1}\right)\right)$, or the relevant information is interpolated from the closest two entries. We use the following formula to compute the unexercised option value $C_{a, t}^{\mathrm{go}}(m, k)$ :

$$
C_{a, t}^{\mathrm{go}}(m, k)=e^{-r_{f}} \sum_{j^{1}=-n}^{n} P^{1}\left(j^{1}\right) \sum_{j^{2}=-n}^{n} P^{2}\left(j^{2}\right) C^{\text {interp }}(m) .
$$

Denote the exercised value of the claim by $C_{t}^{a \text { stop }}(m, k)$. For a two-asset American strangle option with strikes $K_{1}$ and $K_{2}$, $K_{1}<K_{2}$,

$$
\begin{aligned}
C_{a, t}^{\text {stop }}(m, k)=\max \{ & {\left[K_{1}-\max \left(S_{t}^{1}, S_{t}^{2}\right)\right]^{+}, } \\
& {\left.\left[\max \left(S_{t}^{1}, S_{t}^{2}\right)-K_{2}\right]^{+}\right\} . }
\end{aligned}
$$

\begin{tabular}{|c|c|c|c|}
\hline$T$ & $n$ & Option prices with lattice & $\begin{array}{c}\text { Option prices and intervals } \\
\text { with LSM }\end{array}$ \\
\hline \multirow{5}{*}{5} & 1 & 0.014987254820724 & \multirow{5}{*}{$\begin{array}{l}0.029606167588959 \\
{\left[\begin{array}{ll}0.02804 & 0.03117\end{array}\right]}\end{array}$} \\
\hline & 2 & 0.030190542190626 & \\
\hline & 4 & 0.030393224336490 & \\
\hline & 7 & 0.030981333835160 & \\
\hline & 10 & 0.031863879158063 & \\
\hline \multirow{5}{*}{7} & 1 & 0.068487810335143 & \multirow{5}{*}{$\begin{array}{l}0.081499912354419 \\
{\left[\begin{array}{ll}0.07999 & 0.08500\end{array}\right]}\end{array}$} \\
\hline & 2 & 0.081254039347033 & \\
\hline & 4 & 0.083605936631716 & \\
\hline & 7 & 0.081680163494625 & \\
\hline & 10 & 0.080301350887482 & \\
\hline \multirow{5}{*}{10} & 1 & 0.178948896057298 & \multirow{5}{*}{$\begin{array}{l}0.193857807991106 \\
{\left[\begin{array}{ll}0.18842 & 0.19929\end{array}\right]}\end{array}$} \\
\hline & 2 & 0.192329465764766 & \\
\hline & 4 & 0.195314383716280 & \\
\hline & 7 & 0.199868029599249 & \\
\hline & 10 & 0.193058780881166 & \\
\hline
\end{tabular}

The value of the claim at the $k$ th entry of node $(t, m)$ is then

$$
C_{a, t}(m, k)=\max \left\{C_{a, t}^{\mathrm{go}}(m, k), C_{a, t}^{\mathrm{stop}}(m, k)\right\} .
$$

The final option price, obtained by backward recursion of this procedure, is given by $C_{a, 0}(0,1)$.
TABLE 2: Numerical results for Example 1.

\section{Numerical Examples}

In this section, several examples are implemented using the lattice method in this paper and least square Monte-Carlo method (LSM) developed by Longstaff and Schwartz [14].

In Examples 1, 2, and 3, we focus on the single asset American strangle options under GARCH model where the convergence with respect to $n$ and $K$ are studied, respectively, in the first two examples, and the optimal exercise boundaries are drawn for the third example. In Examples 4 and 5, we compute the two-dimensional assets American strangle options.

In Tables 2 and 3, the prices of the options using LSM with 5,000 paths are calculated and the intervals that the true prices fall into are provided. From the comparisons we confirm that the lattice methods developed in this paper are correct and reliable. Furthermore from Table 2 we observe that the lattice method converges as $n$ goes larger and from Table 3 the lattice method converges as $K$ goes larger. Figure 2 shows exercise and holding regions: the middle part is the holding region and the top and bottom parts are the exercise regions.

Example 1. Consider single asset GARCH model with parameters $r_{f}=5 \%, q=10 \%, \beta_{0}=6.575 \times 10^{-6}, \beta_{1}=0.9$, $\beta_{2}=0.04, S_{0}=100, h_{0}=0.0001096, K_{1}=105, K_{2}=95$, $\gamma=h_{0}$, and $c^{*}=0$. Fixing $K=20$, we investigate the convergence behavior as $n$ increases.

Example 2. Consider single asset GARCH model with the same parameters as Example 1. In this example, $n=5$ and the sensitivity to the volatility space parameter, $K$, is explored.

Example 3. Consider single asset GARCH model with the same parameters as Example 1. Draw the figure of the optimal exercise boundaries for American strangle. 
TABLE 3: Numerical results for Example 2.

\begin{tabular}{|c|c|c|c|}
\hline$T$ & K & Option prices with lattice & $\begin{array}{c}\text { Option prices and intervals } \\
\text { with LSM }\end{array}$ \\
\hline \multirow{6}{*}{5} & 2 & 0.028187456298155 & \multirow{6}{*}{$\begin{array}{l}0.029606167588959 \\
{\left[\begin{array}{ll}0.02804 & 0.03117\end{array}\right]}\end{array}$} \\
\hline & 4 & 0.029090524706680 & \\
\hline & 6 & 0.029930565933099 & \\
\hline & 10 & 0.030190542190626 & \\
\hline & 20 & 0.029391074539888 & \\
\hline & 40 & 0.029489977773055 & \\
\hline \multirow{6}{*}{10} & 2 & 0.197070210662258 & \multirow{6}{*}{$\begin{array}{l}0.193857807991106 \\
{\left[\begin{array}{ll}0.18842 & 0.19929\end{array}\right]}\end{array}$} \\
\hline & 4 & 0.195700639175277 & \\
\hline & 6 & 0.196465071653130 & \\
\hline & 10 & 0.192329465764766 & \\
\hline & 20 & 0.193696054251151 & \\
\hline & 40 & 0.194423204241922 & \\
\hline \multirow{6}{*}{30} & 2 & 1.182560819901510 & \multirow{6}{*}{$\begin{array}{l}1.205198709585444 \\
{\left[\begin{array}{ll}1.19372 & 1.22670\end{array}\right]}\end{array}$} \\
\hline & 4 & 1.197816540629624 & \\
\hline & 6 & 1.200350772727828 & \\
\hline & 10 & 1.202714267674711 & \\
\hline & 20 & 1.203407511987706 & \\
\hline & 40 & 1.203545193979154 & \\
\hline
\end{tabular}

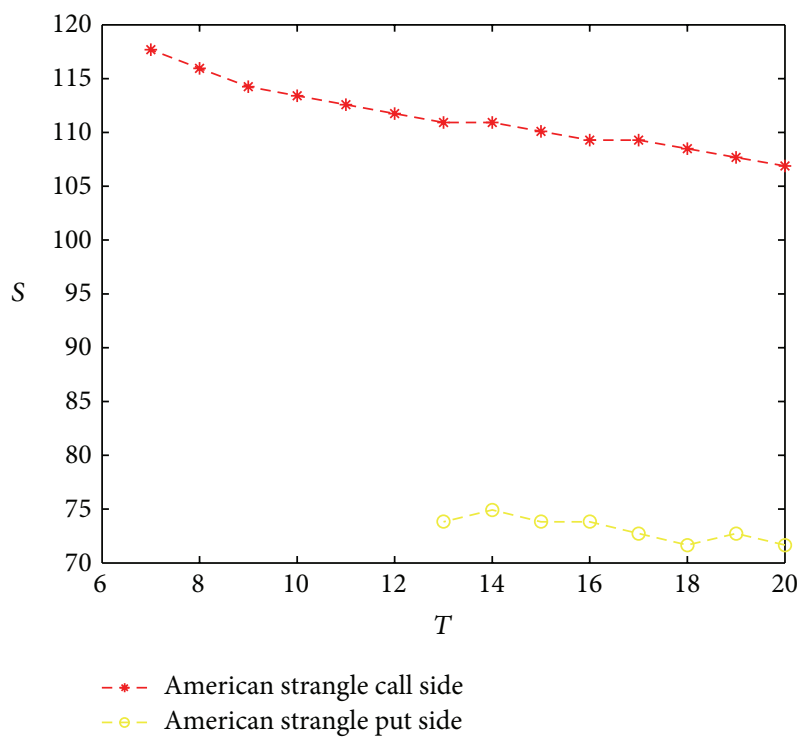

Figure 2: Optimal exercise boundaries for Example 3.

In Examples 4 and 5, we examine the stochastic lattice methods for pricing American strangle options under multiasset under stochastic volatility model where the convergence with $n$ and $K$ are studied. From the numerics in Tables 4 and 5 , we confirm that the lattice methods for two-dimensional models are correct and reliable and the convergence of the lattice methods with respect to $n$ and $K$ is observed.

Example 4. Consider two-asset American strangles with param- eters $r_{f}=5 \%, q^{1}=q^{2}=10 \%, \beta_{0}^{1}=\beta_{0}^{2}=6.575 \times 10^{-6}$, $\beta_{1}^{1}=\beta_{1}^{2}=0.9, \beta_{2}^{1}=\beta_{2}^{1}=0.04, S_{0}^{1}=S_{0}^{2}=100$,
TABLE 4: Numerical results for Example 4.

\begin{tabular}{|c|c|c|c|}
\hline$T$ & $n$ & Option prices with lattice & $\begin{array}{c}\text { Option prices and interval } \\
\text { with LSM }\end{array}$ \\
\hline \multirow{5}{*}{3} & 1 & 0.005974297 & \multirow{5}{*}{$\begin{array}{l}0.005065587636226 \\
{\left[\begin{array}{ll}0.00261 & 0.006316\end{array}\right]}\end{array}$} \\
\hline & 2 & 0.004223692 & \\
\hline & 3 & 0.004502351 & \\
\hline & 4 & 0.005530355 & \\
\hline & 5 & 0.005040507 & \\
\hline \multirow{5}{*}{4} & 1 & 0.014427788 & \multirow{5}{*}{$\begin{array}{l}0.015018277749022 \\
{\left[\begin{array}{ll}0.01111 & 0.01892\end{array}\right]}\end{array}$} \\
\hline & 2 & 0.014590056 & \\
\hline & 3 & 0.013984718 & \\
\hline & 4 & 0.017223364 & \\
\hline & 5 & 0.015988485 & \\
\hline \multirow{5}{*}{5} & 1 & 0.022129134 & \multirow{5}{*}{$\begin{array}{l}0.034841312405081 \\
{\left[\begin{array}{ll}0.02844 & 0.041235\end{array}\right]}\end{array}$} \\
\hline & 2 & 0.030507636 & \\
\hline & 3 & 0.030853276 & \\
\hline & 4 & 0.035594538 & \\
\hline & 5 & 0.034694665 & \\
\hline
\end{tabular}

TABLe 5: Numerical results for Example 5.

\begin{tabular}{|c|c|c|c|}
\hline$T$ & $K$ & Option prices with lattice & $\begin{array}{c}\text { Option prices and intervals } \\
\text { with LSM }\end{array}$ \\
\hline \multirow{5}{*}{3} & 2 & 0.005974297 & \multirow{5}{*}{$\begin{array}{l}0.005065587636226 \\
{\left[\begin{array}{ll}0.00261 & 0.006316\end{array}\right]}\end{array}$} \\
\hline & 4 & 0.005974297 & \\
\hline & 6 & 0.005974297 & \\
\hline & 8 & 0.005974297 & \\
\hline & 10 & 0.005974297 & \\
\hline \multirow{5}{*}{4} & 2 & 0.014427788 & \multirow{5}{*}{$\begin{array}{l}0.015018277749022 \\
{\left[\begin{array}{ll}0.01111 & 0.01892\end{array}\right]}\end{array}$} \\
\hline & 4 & 0.014427788 & \\
\hline & 6 & 0.014427788 & \\
\hline & 8 & 0.014427788 & \\
\hline & 10 & 0.014427788 & \\
\hline \multirow{5}{*}{5} & 2 & 0.02194509 & \multirow{5}{*}{$\begin{array}{l}0.034841312405081 \\
{\left[\begin{array}{ll}0.02844 & 0.041235\end{array}\right]}\end{array}$} \\
\hline & 4 & 0.022129134 & \\
\hline & 6 & 0.022164226 & \\
\hline & 8 & 0.024355984 & \\
\hline & 10 & 0.024276964 & \\
\hline
\end{tabular}

$h_{0}^{1}=h_{0}^{2}=0.0001096, K_{1}=105, K_{2}=95, \gamma^{1}=\gamma^{2}=h_{0}^{1}$, and $c^{*, 1}=c^{*, 2}=0$. Fixing $K=4$, we investigate the convergence behavior as $n$ increases.

Example 5. Consider the two-dimensional GARCH model with the same parameters as Example 4. Fixing $n=1$, we study the sensitivity to the volatility space parameter $K$.

\section{Conclusions}

In this paper we studied pricing methods for stochastic volatility models of the American strangles with single asset and multiassets. Both lattice methods and LSM methods are developed and implemented. To the best of our knowledge, 
there are no results on the lattice methods for multidimensional stochastic volatility models. We first extended the stochastic lattice methods invented by Ritchken and Trevor [2] which are for one-dimensional GARCH models of American call to the multidimensional GARCH models of American strangles. Numerical examples confirm the correctness and reliability of the lattice methods. Future challenging works include the development of the lattice methods for multidimensional volatility models with correlations and recently developed models (e.g., [15]). One possible solution to the case of correlation is to adopt the idea (using momentgenerating function) in [7]. However it needs to develop new techniques when the stochastic volatility models are involved. Furthermore, a dimensional-reduction technique should be developed to reduce the computational cost.

\section{Conflict of Interests}

The authors declare that there is no conflict of interests regarding the publication of this paper.

\section{Acknowledgment}

The work was supported by the Fundamental Research Funds for the Central Universities (Grant no. JBK130401).

\section{References}

[1] S. L. Heston, "A closed-form solution for options with stochastic volatility with applications to bond and currency options," Review of Financial Studies, vol. 6, pp. 327-343, 1993.

[2] P. Ritchken and R. Trevor, "Pricing options under generalized GARCH and stochastic volatility processes," Journal of Finance, vol. 54, no. 1, pp. 377-402, 1999.

[3] N. Cakici and K. Topyan, "The GARCH option pricing model: a lattice approach," Quantitative Finance, vol. 2, pp. 432-442, 2000.

[4] C.-C. Wu, "The GARCH option pricing model: a modification of lattice approach," Review of Quantitative Finance and Accounting, vol. 26, no. 1, pp. 55-66, 2006.

[5] E. Akyildirim, Y. Dolinsky, and H. Soner, "Approximating stochastic volatility by recombinant trees," http://arxiv.org/abs/ $1205.3555 \mathrm{v} 1$.

[6] P. Boyle, "A lattice framework for option pricing with two state variables," Journal of Financial and Quantitative Analysis, vol. 23, pp. 1-2, 1988.

[7] P. Boyle, J. Evnine, and S. Gibbs, "Numerical evaluation of multivariate contingent claims," Review of Financial Studied, vol. 2, pp. 241-250, 1989.

[8] R.-R. Chen, S.-L. Chung, and T. T. Yang, "Option pricing in a multi-asset, complete market economy," Journal of Financial and Quantitative Analysis, vol. 37, no. 4, pp. 649-666, 2002.

[9] A. Gamba and L. Trigeorgis, "An improved binomial lattice method for multi-dimensional options," Applied Mathematical Finance, vol. 14, no. 5, pp. 453-475, 2007.

[10] K.-S. Moon, W.-J. Kim, and H. Kim, "Adaptive lattice methods for multi-asset models," Computers \& Mathematics with Applications, vol. 56, no. 2, pp. 352-366, 2008.
[11] C. Chiarella and A. Ziogas, "Evaluation of American strangles," Journal of Economic Dynamics \& Control, vol. 29, no. 1-2, pp. 31-62, 2005.

[12] F. Moraux, "On perpetual American strangles," Journal of Derivatives, vol. 16, no. 4, pp. 82-97, 2009.

[13] J.-C. Duan, “The GARCH option pricing model," Mathematical Finance, vol. 5, no. 1, pp. 13-32, 1995.

[14] F. A. Longstaff and E. S. Schwartz, "Valuing American options by simulation: a simple least-squares approach," Review of Financial Studies, vol. 14, no. 1, pp. 113-147, 2001.

[15] C. X. Huang, X. Gong, X. Chen, and F. H. Wen, "Measuring and forecasting volatility in Chinese stock market using HAR-CJM model," Abstract and Applied Analysis, vol. 2013, Article ID 143194, 13 pages, 2013. 


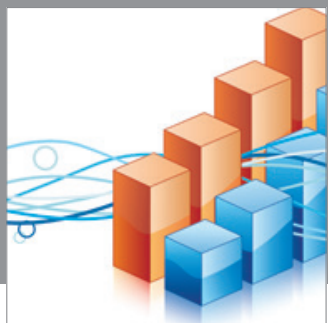

Advances in

Operations Research

mansans

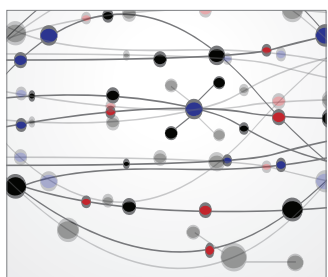

The Scientific World Journal
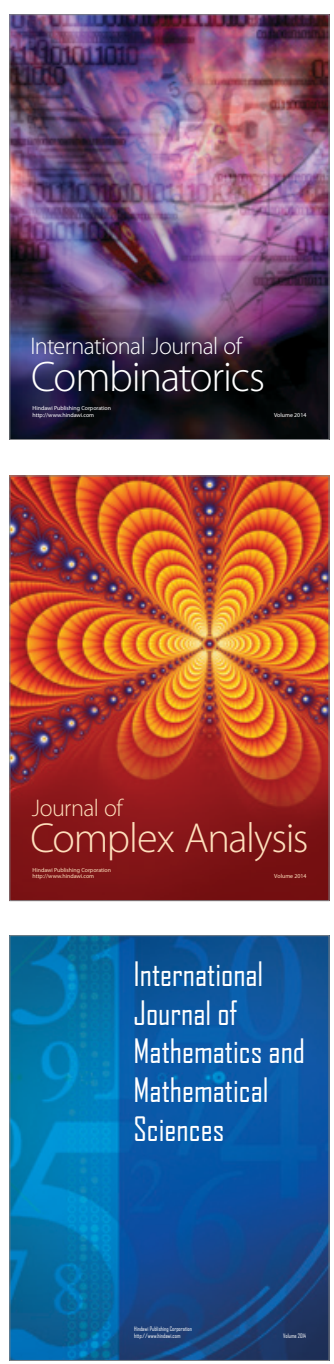
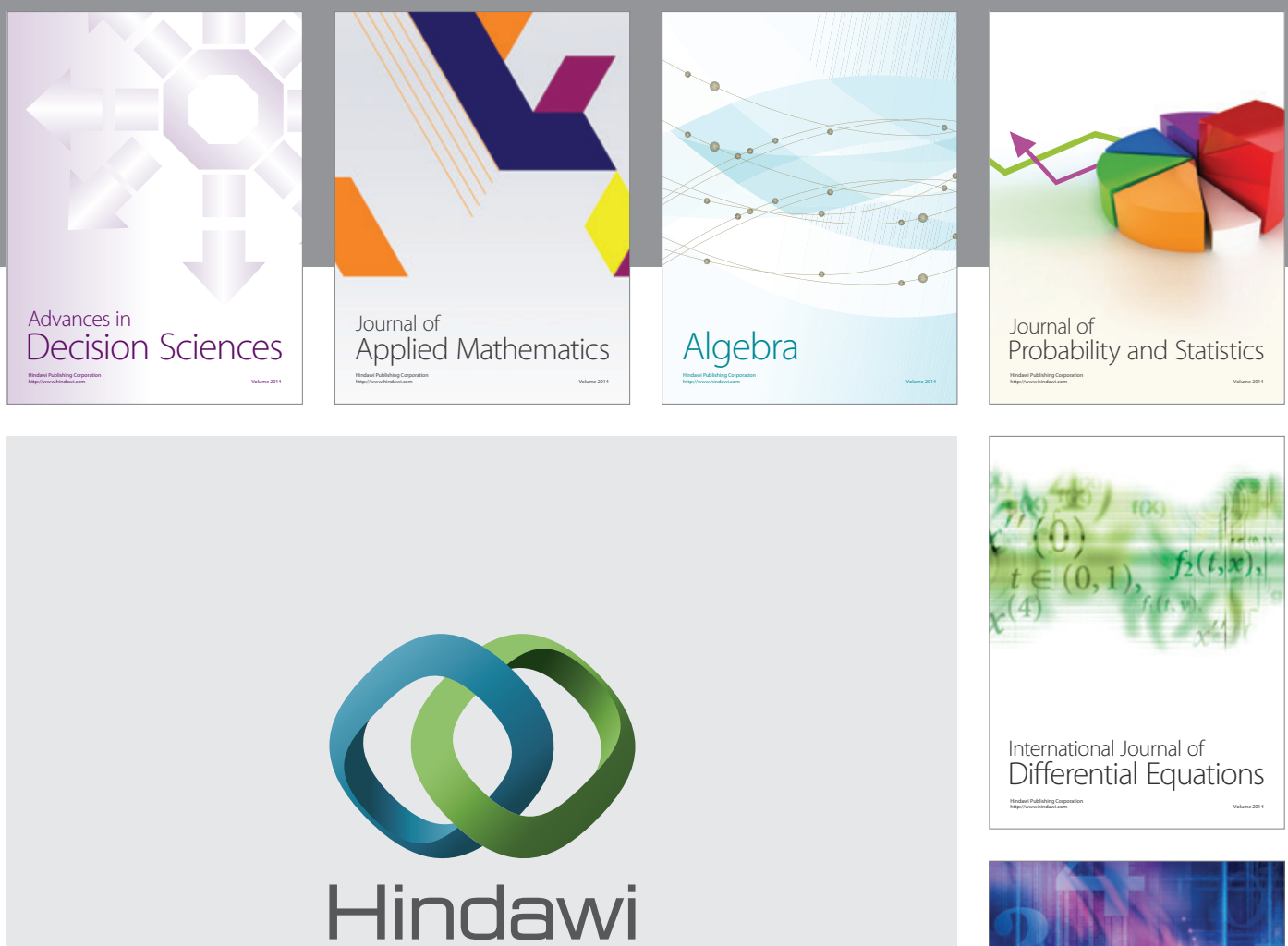

Submit your manuscripts at http://www.hindawi.com
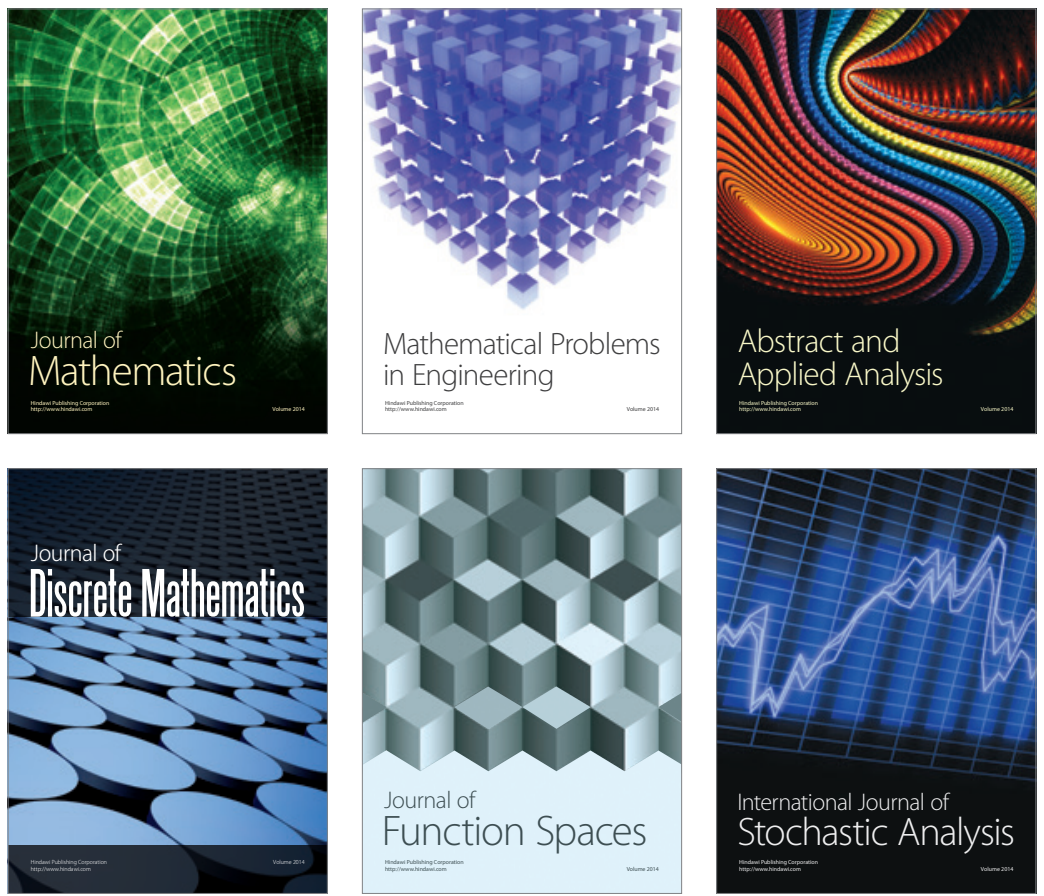

Journal of

Function Spaces

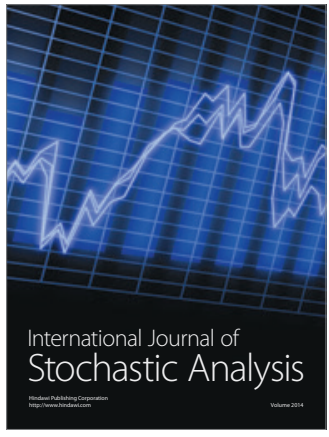

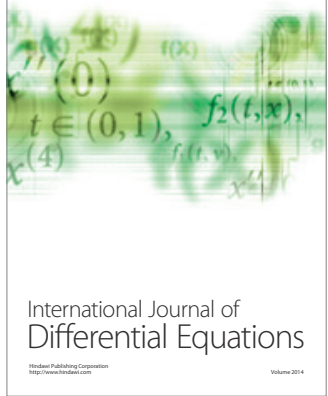
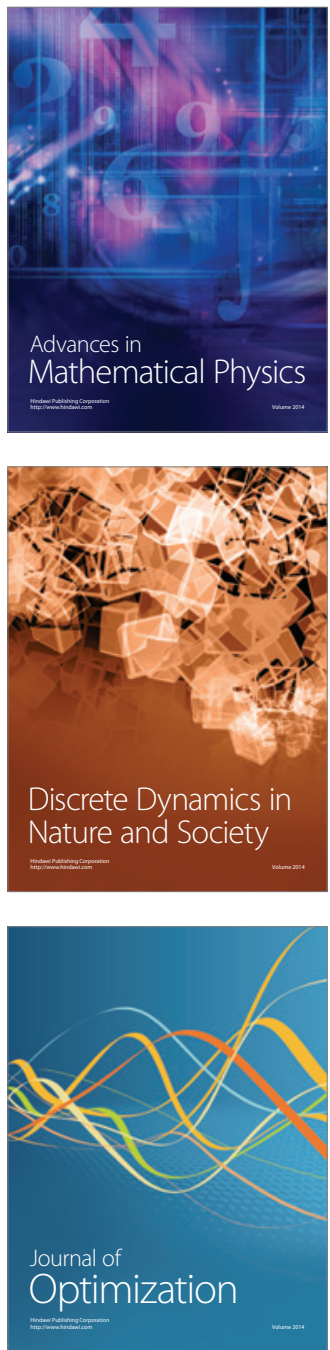